\title{
PEG 8000 increases solubility and dissolution rate of quercetin in solid dispersion system
}

\author{
Setyawan Dwi 1, *, Setiawardani Febrianti ${ }^{1}$, Amrullah Zainul ${ }^{1}$, Sari Retno ${ }^{1}$ \\ 1 Department of Pharmaceutics, Faculty of Pharmacy, Airlangga University, Surabaya 60286, Indonesia \\ * Correspondence: dwisetyawan-90@ff.unair.ac.id (S.D.); Tel: +62-31-5033710; ORCID No: 0000-0001-8009-6054.
}

Received: 6 September 2017 / Revised: 29 October 2017 / Accepted: 30 October 2017

\begin{abstract}
The aim of this study was to examine the physical characteristics, solubility, and in vitro dissolution profile of quercetin-PEG 8000 solid dispersion prepared by melting method. Solid dispersion was prepared by melting method with various ratios of quercetin and PEG 8000 (1:1; 1:2; 1:3). The solid dispersion was characterized using differential thermal analyzer (DTA), powder X-ray diffraction (PXRD), scanning electron microscope (SEM), and fourier transform infrared spectroscopy (FTIR). The solubility and in vitro dissolution of quercetin-PEG 8000 solid dispersion were evaluated and compared to physical mixture and pure quercetin. The solid dispersion of quercetin-PEG 8000 has lower melting point and crystal peak intensity compared to pure quercetin and physical mixture. The infrared spectra of solid dispersions and physical mixtures indicated the occurrence of physical interactions that involved weak bonding forces such as the hydrogen bond between quercetin functional groups and O-H group. The solubility and the dissolution rate enhanced in accordance with increasing of PEG 8000 amount.
\end{abstract}

KEYWORDS: Quercetin; PEG 8000; solid dispersion; solubility; dissolution rate.

\section{INTRODUCTION}

Quercetin is a flavonols, one of six subclasses of flavonoids containing high antioxidants that can be used as potent a chemopreventive and can be used a strong barrier to the growth of breast, colon, lung, and ovarian cancer cells [1]. One of the problems quercetin is classified as Biopharmaceutics Classification System (BCS) II, which means that it exhibits high permeability but low solubility, thus affecting the bioavailability [2]. Quercetin consists of five hydroxyl groups that determine its biological activity. However, the quercetin molecule is lipophilic or water-insoluble [3]. It is known that quercetin has low solubility at room temperature and at $\mathrm{pH} 3 \mathrm{of} 0.4 \mathrm{\mu g} / \mathrm{mL}$ [4]. Therefore, the bioavailability of quercetin is very low at about $1 \%$ in humans and less than $17 \%$ in rats. Quercetin is rapidly metabolized and $99.4 \%$ attached to plasma proteins [5]. Quercetin is now marketed as a dietary supplement with a recommended daily dose of 200-1200 mg [6]. Quercetin is a stable flavonoid because of its high melting point around $326^{\circ} \mathrm{C}$ [4].

Various methods have been used to increase the solubility of active pharmaceutical ingredients (API). The solid dispersion method is commonly employed to increase the dissolution rate and the bioavailability of low-solubility drugs due to its simple, cost-effective, and beneficial characteristics [7]. Solid dispersion is a promising technique for improving the absorption and bioavailability of oral administration routes for medicinal ingredients belonging to the second-class biopharmaceutical classification system [9]. In general, solid dispersion consists of two distinct components were hydrophobic drug and hydrophilic carrier matrix [8]. The solid dispersion may increase the solubility of the drug by means amorphous crystals, which increase the surface area of the drug substances by the diminution of the particle size. Thus, the drug substance it has a greater chance of being contacted with the medium and the interfacial stress is lowered by the water-soluble carrier [10]. In addition, the solid dispersion increases the wetting and increases the porosity degree so as to increase the solubility of the drug substance [9].

Polyethylene glycol 8000 (PEG 8000) is one of matrixes used in solid dispersion formation. PEG 8000 is known to exhibit hydrophilic, non-toxic, and non-irritant characteristics. PEG with molecular weights of 1500 to 20.000 are usually used to form a solid dispersion system. PEG with the higher molecular weight exhibits a higher viscosity. In the present study, PEG 8000 was used because it performs a high water-solubility, a good stability in room temperature and a non-hygroscopic feature. The relatively low melting point, around $60^{\circ} \mathrm{C}$,

How to cite this article: Dwi S, Febrianti S, Zainul A, Retno S. PEG 8000 increases solubility and dissolution rate of quercetin in solid dispersion system. Marmara Pharm J. 2018; 22 (2): 259-266.

(C) 2018 Marmara University Press

ISSN: 1309-0801 
is an advantageous property for manufacture of solid dispersion by the melting method. It has been reported that a solid dispersion using PEG 8000 increases the solubility of poorly soluble substances such as albendazole and gliclazide [11, 12].

\section{RESULTS AND DISCUSSION}

\subsection{Thermal Analysis Results using DTA}

The thermogram comparison of quercetin hydrate (Q), PEG 8000 (PEG) physical mixture of quercetin PEG 8000 1:1 (PM1), physical mixture quercetin - PEG 8000 1:2 (PM2), physical mixture quercetin - PEG 8000 1:3 (PM3), quercetin solid dispersion - PEG 8000 1:1 (SD1), quercetin solid dispersion - PEG 8000 1:2 (SD2), and quercetin solid dispersion - PEG 8000 1:3 (SD3) was shown in Figure 1.

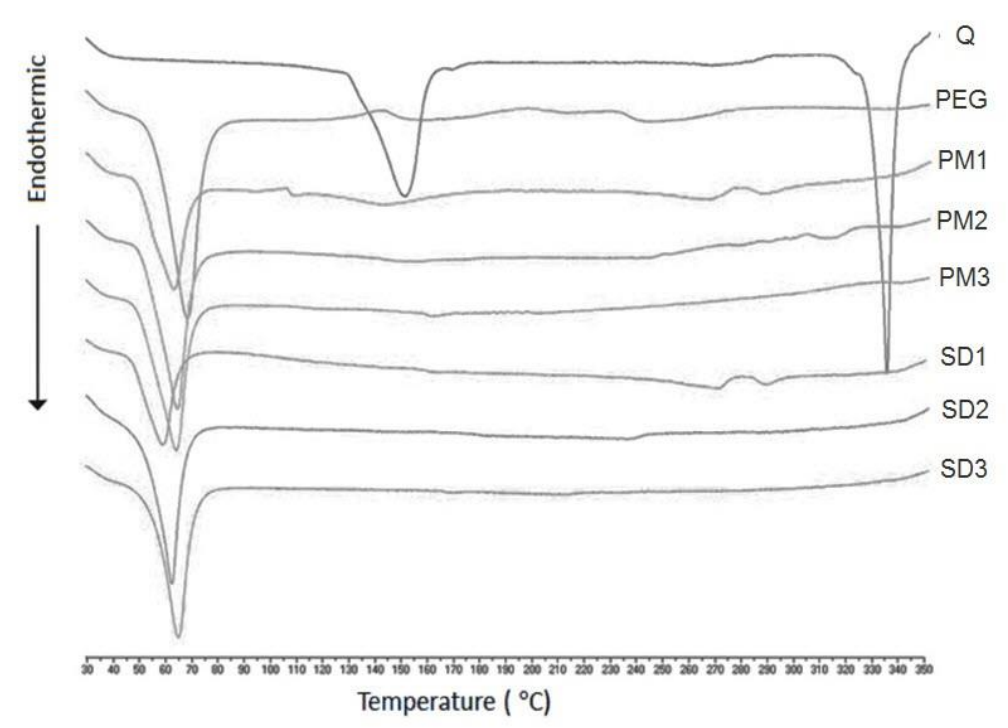

Figure 1.Thermogram DTA Q, PEG, PM1,PM2, PM3, SD1, SD2 and SD3.

Quersetin has an endothermic peak at $325.4{ }^{\circ} \mathrm{C}$ indicates that quercetin is in crystalline form. It is in accordance with the reference that quercetin has melting point at $326^{\circ} \mathrm{C}$ [4]. The endothermic event at a 150$160{ }^{\circ} \mathrm{C}$ is possibly caused by the release of water molecules from quercetin dihydrate. The thermogram of physical mixture and solid dispersion of quercetin-PEG8000 with a ratio of 1:1 appears to have three endothermic peaks at $61.2^{\circ} \mathrm{C}, 262.9^{\circ} \mathrm{C}$, and $282.9^{\circ} \mathrm{C}$ that indicates the appearance of PEG 8000 endothermic peak and the shift of quercetin endothermic peak to a lower temperature. The thermogram of physical mixture quercetin-PEG 8000 with ratio $1: 2$ shows two endothermic peaks at $62.1^{\circ} \mathrm{C}$ and $306.6^{\circ} \mathrm{C}$, whereas the solid dispersion has only one endothermic peak at a melting temperature of $60.5^{\circ} \mathrm{C}$. The dissapearance of the endothermic peak indicates that quercetin has dispersed molecularly within the PEG matrix. Both of the physical mixture and solid dispersion with ratio of 1:3 has not shown any quercetin endothermic. This endothermic peak decline indicates crystallinity quercetin-PEG solid dispersion toward the amorphous $[5,9]$.

\subsection{Characterization using Powder X-ray Diffraction Method (PXRD)}

The diffractogram at Figure 2 shows that quercetin is in crystalline and PEG 8000 is in semicrystalline form demonstrated by two high-intensity diffractogram peaks at $2 \theta=19.12^{\circ}$ and $2 \theta=23.25^{\circ}$. The observation of the intensity of the difractogram at $2 \theta$ indicates that the solid dispersion underwent a decrease in intensity as compared to pure quercetin and the physical mixture. This may be due to the lipophilic quercetin even though that has 5- hydroxyl groups and PEG 8000 is hydrophilic. Therefore, although quercetin is dispersed in the polymer, there is a tendency to bond between the quercetin molecules themselves which leads to the growth of quercetin crystals [3]. 


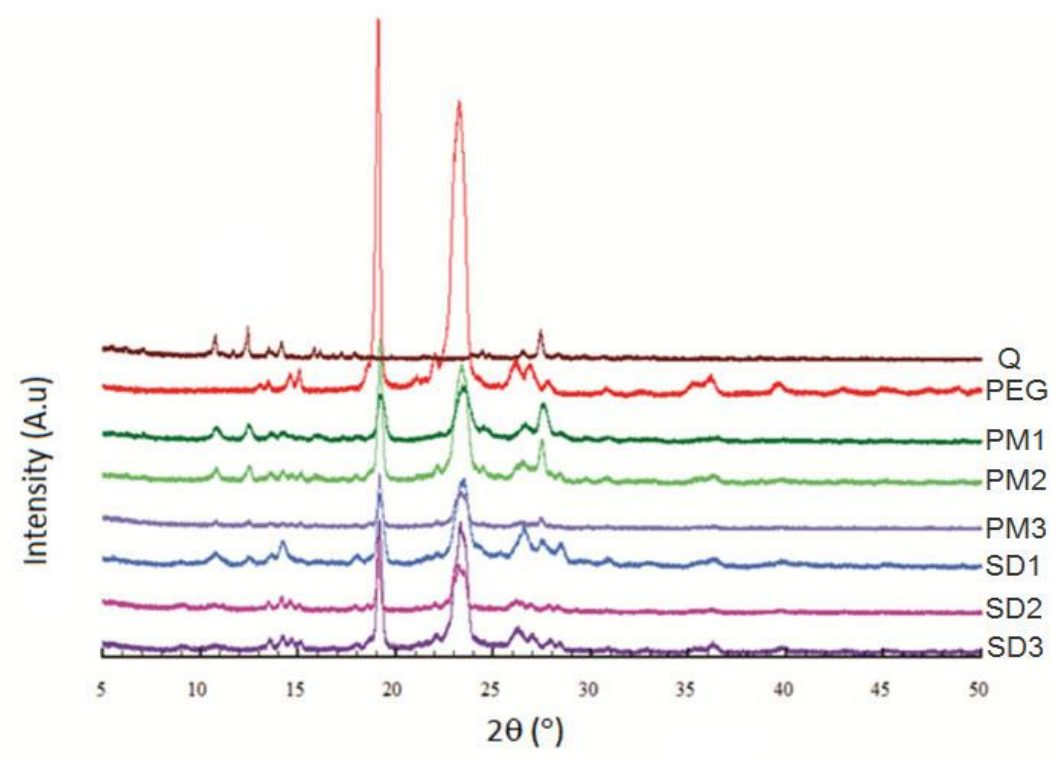

Figure 2. Diffractogram X-ray of Q, PEG, PM1, PM2, PM3, SD1, SD2, and SD3.

\subsection{Characterization using Fourier Transform Infrared Spectroscopy}

The characterization using FTIR spectroscopy is shown in Figure 3. The IR profile of quercetin showed specific band at $3340 \mathrm{~cm}^{-1}$ demonstrating free $-\mathrm{OH}$ bond vibration, strong doublet at $1660 \mathrm{~cm}^{-1}$ and $1656 \mathrm{~cm}^{-1}$ representing $\mathrm{C}=\mathrm{O}$ group stretching, band at $1510 \mathrm{~cm}^{-1}$ for aromatic group, bands at $1310 \mathrm{~cm}^{-1}$ and $1160 \mathrm{~cm}^{-1}$ for C-O-C group vibration, band at $999 \mathrm{~cm}^{-1}$ for aromatic ring-bonded $\mathrm{C}-\mathrm{H}$, and bands at $2880 \mathrm{~cm}^{-1}$ and 1110 $\mathrm{cm}^{-1}$ for C-H stretching and C-O (ether) stretching [5].

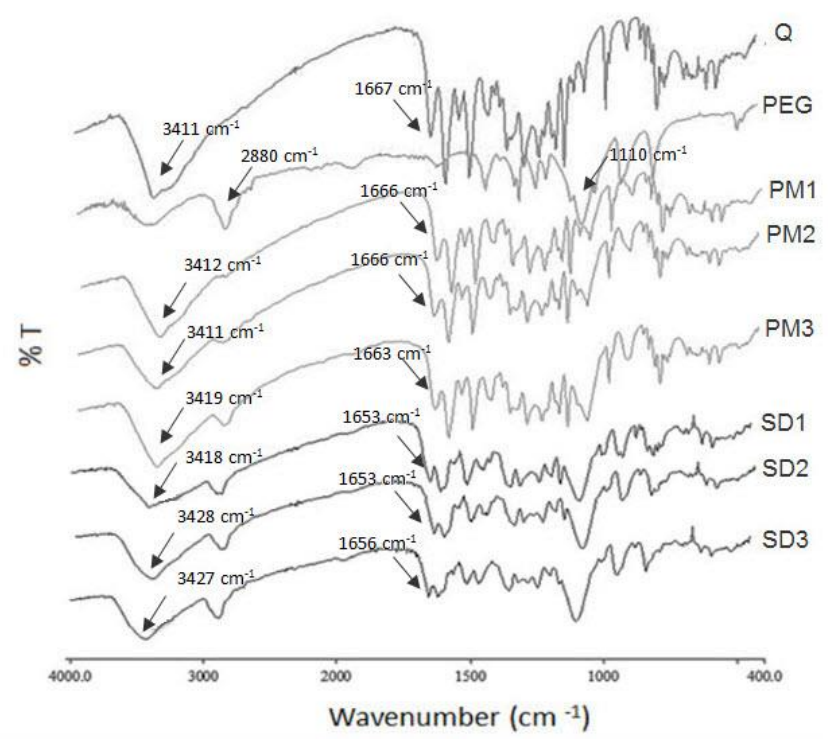

Figure 3. Infrared spectrogram of Q, PEG, PM1, PM2, PM3, SD1, SD2, and SD3.

We found that there was a shift in carbonyl group peak from $1666 \mathrm{~cm}^{-1}$ to $1656 \mathrm{~cm}^{-1}$ in solid dispersion system suggesting the possibility of hydrogen bond between carbonyl group from quercetin and terminal hydroxyl group from PEG $8000[13,14]$.

The absorption band shift occurs to $3418 \mathrm{~cm}^{-1}$ for SD1, $3428 \mathrm{~cm}^{-1}$ for SD2, and $3427 \mathrm{~cm}^{-1}$ for SD3. The $\mathrm{C}=\mathrm{O}$ quercetin group has an absorption band at a wavenumber of $1667 \mathrm{~cm}^{-1}$ and $1612 \mathrm{~cm}^{-1}$. The absorption band shift occured to a wavenumber of $1653 \mathrm{~cm}^{-1}$ and $1613 \mathrm{~cm}^{-1}$ for SD1, $1653 \mathrm{~cm}^{-1}$ and $1616 \mathrm{~cm}^{-1}$ for SD2, and $1656 \mathrm{~cm}^{-1}$ and $1616 \mathrm{~cm}^{-1}$ for SD3. 
This shift of infrared spectra wavenumber indicates a physical interaction involving weak bonds such as hydrogen bonds between functional groups on quercetin and -OH groups in PEG 8000 . The presence of a hydrogen bond does not alter the chemical properties of quercetin. Previous study demonstrates that quercetin-PEG interaction in solid dispersion system is also indicated by broadening and shifting of hydroxyl vibration band of PEG from 3800 to $3000 \mathrm{~cm}^{-1}$ [13]. Solid dispersion was developed in solid state. Thus, it is difficult to charactherize solid dispersion using NMR analysis because the sample should be diluted before analysis.

\subsection{Characterization using Scanning Electron Microscopy (SEM)}

The results of morphological observations on crystal habit of quercetin (Q), PEG 8000 (PEG), and quercetin solid dispersion - PEG 8000 1: 3 (SD3) were shown in Figure 4. The surface morphology of the samples observed using SEM showed $Q$ dispersing in the PEG polymer. Photomicrograph shows that quercetin crystals have irregular flake shape with needle-like fragments [5] in Figure 4A, while PEG 8000 appears to be in large pieces, as previously reported Figure 4B [9].

A solid dispersion ratio of 1:3 shows PEG particles forming a solid aggregate and only slightly visible small quercetin crystals dispersed within the PEG matrix. This quercetin-PEG 8000 SD includes solid dispersion type glass transition where the drug substance or quercetin is dispersed in the form of crystalline particles in the PEG 8000 matrix. Stability test is also considered to be important test to measure how long and in what condition the solid dispersion produced is subjected to recrystallization. Further examination is needed to clarify the stability of quercetin-PEG 8000 solid dispersion systems.
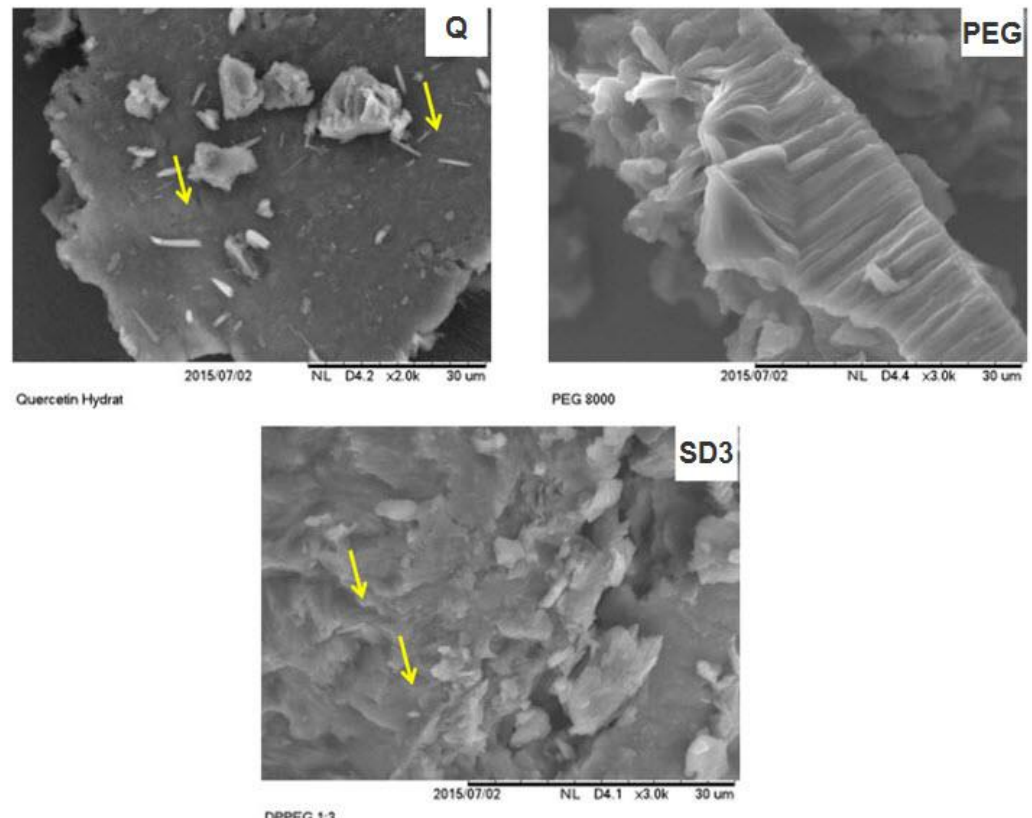

Figure 4. Photographs SEM of Q, PEG and SD3, 2000X magnification.

\subsection{Solubility test}

Saturated solubility of quercetin is to $1.62 \pm 0.12 .10^{-4}(\%)$ in a medium buffer citric acid $-\mathrm{NaOH}$ pH 5.0 \pm 0.5 at temperature of $30 \pm 0.5^{\circ} \mathrm{C}$ (Figure 5). Meanwhile, the solubility of quercetin in the previous study was 7.0.10-4 (\%) in water [15]. Media buffer $\mathrm{pH} 5.0 \pm 0.5$ is used as a medium because quercetin is stable at this $\mathrm{pH}$ [16]. Quercetin solubility of 1:1 physical mixture was $1.15 \pm 0.25 .10^{-4}(\%), 1: 2$ physical mixture was $1.34 \pm 0.20 .10^{-}$ ${ }^{4}(\%)$ and $1: 3$ physical mixture was $0.95 \pm 0.02 \cdot 10^{-4}(\%)$. Meanwhile, the solubility test results for $1: 1$ solid dispersion was $2.01 \pm 0.19 .10^{-4}(\%), 1: 2$ solid dispersion was $2.43 \pm 0.11 .10^{-4}(\%)$ and $1: 3$ solid dispersion was $5.26 \pm 0.43 \cdot 10^{-4}(\%)$. The data indicates that there is an increase in the solubility of quercetin when prepared in a solid dispersion system with PEG 8000 polymer compared to a physical and pure quercetin. The solubility of quercetin performed by quercetin solid dispersion system - PEG 8000 in 1:3 ratio was 3.25 times higher as compared to pure quercetin. 


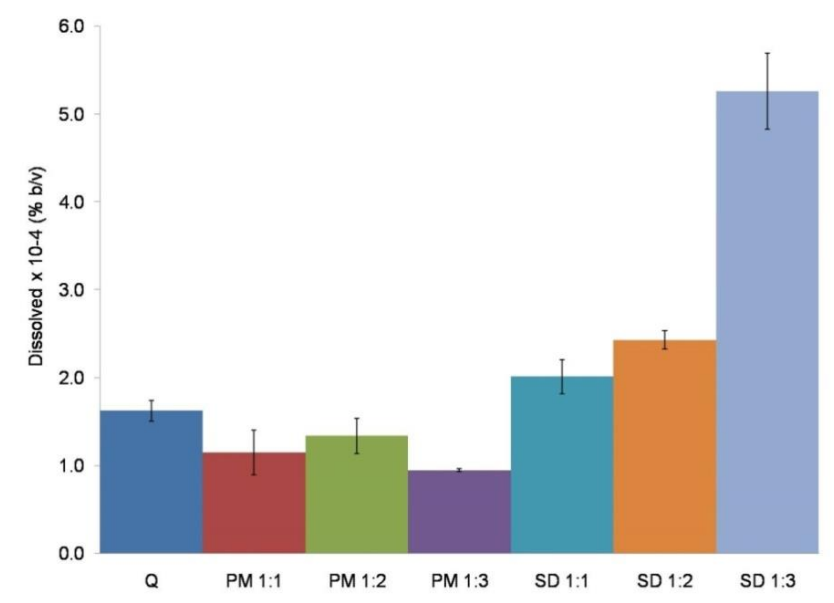

Figure 5. Solubility in buffer citric acid - $\mathrm{NaOH}$ pH $5.0 \pm 0.5$ medium, at a $30 \pm 0.5^{\circ} \mathrm{C}$.

The results of one-way ANOVA statistical analysis showed that the solubility of 1:3 solid dispersion demonstrated $\mathrm{p}$ value $<0.05$. This indicates a significant difference in the solubility between quercetin and 1:3 solid dispersion. The solubility of quercetin has been increased by the formation of solid dispersion system, possibly due to physical changes that decrease the particle size [17]. Decreased particle size can increase the area of contact with the media. In addition, the solid dispersion system with the ratio of PEG 8000 (1:3) showed the highest increase in solubility. This is possibly due to a higher amount of polymer used in solid dispersion systems may minimize crystallinity which in turn increases its solubility [18].

\subsection{In-vitro Dissolution Test}

In-vitro dissolution test shown in Figure 6. The solubility of quercetin in the medium of citrate buffer pH $5 \pm 0.5$ is very low, which is approximately $1.62 \pm 0.12$. $10^{-4}(\%)$. Therefore, to achieve a sink condition, $1 \%$ SLS surfactant was added to increase the solubility of quercetin. Since the solid dispersion was developed to be an oral dosage form, $1 \%$ SLS was added into dissolution media to provide sink condition during dissolution test. The dissolution efficiency of minute $30\left(\mathrm{DE}_{30}\right)$ was used to determine the dissolution rate of quercetin, physical mixture and solid dispersion. $\mathrm{DE}_{30}$ for $\mathrm{Q}$ was 58.52, PM 1:1 was 64.86, PM 1:2 was 60.36 and physical PM of 1:3 was 66.63. Meanwhile, the value for SD 1:1 was 38.53, SD 1: 2 was 59.48, and SD 1: 3 was 79.03. The results of one way anova statistical analysis tests of $\mathrm{DE}_{30}$ showed thata significant difference in solubility between quercetin and 1:3 solid dispersion ( $\mathrm{p}$ value of $<0.05$ ).

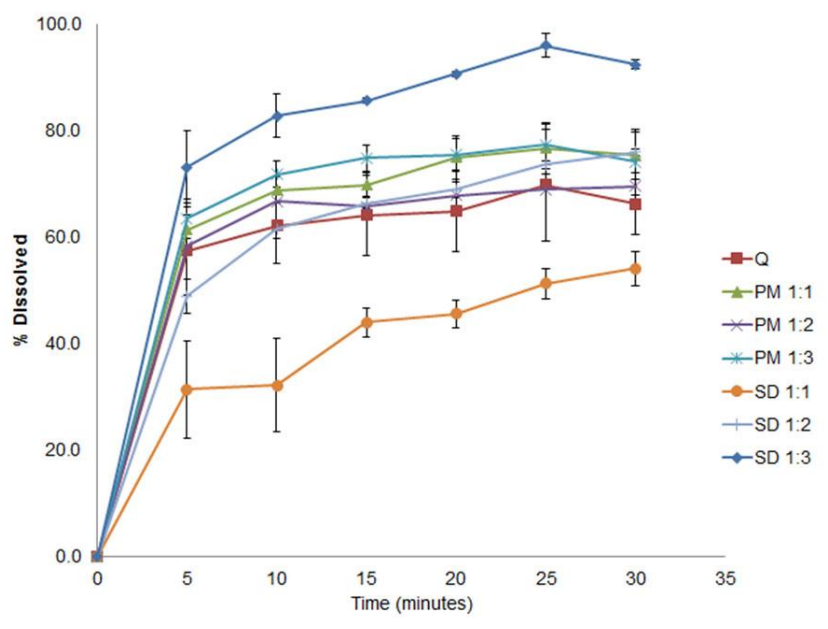

Figure 6. Dissolution profile of Q, PM1, PM2, PM3, SD1, SD2, and SD3 in buffer citric acid - NaOH pH $5.0 \pm$ 0.5 medium + SLS $1 \%$.

The highest increase in quercetin dissolution occured in the 1:3 solid dispersion, it was due to the reduction of particle size so that the surface area of drug contacted with the dissolution media is greater [10]. Moreover, the increase in dissolution may also occur due to an increase in the solubility of quercetin according 
to the Noyes-Whitney equation, i.e. that the solubility of the substance is directly proportional to the rate of dissolution [19]. In addition, since there was also an increase in the rate of dissolution in the physical mixture, it is possible that PEG 8000 polymer in the physical mixture increases the wetting on the surface of the particles [18].

\section{CONCLUSION}

To summarize, the present study shows that the quercetin-PEG 8000 solid dispersion system has been successfully formed, which is shown by the decrease and loss of the endothermic peak of quercetin on the DTA thermogram. The intensity of the x-ray diffractogram is decreased in solid dispersion system. The interaction that occured in solid dispersion systems is only physical interaction involving weak bonds such as hydrogen bonds. Formation of quercetin-PEG 8000 solid dispersion system increased the solubility and in vitro dissolution rate of quercetin as compared to pure quercetin and its physical mixture. Based on the result of the present study, it can be concluded that quercetin in the solid dispersion system with PEG 8000 is dispersed in the amorphous forms which in turn increase the solubility and dissolution rate of quercetin.

\section{MATERIALS AND METHODS}

\subsection{Materials}

The materials used in this study were quercetine hydrate $\left(\mathrm{C}_{15} \mathrm{H}_{10} \mathrm{O}_{7} . \mathrm{xH}_{2} \mathrm{O} ; \mathrm{BM}: 302.24 \mathrm{~g} / \mathrm{mol}\right)$ from Tokyo Chemical Industry Co., LTD, Japan, Lot 83N $2 \mathrm{O}$; polyethylene glycol(PEG) 8000 (Fluka, Switzerland Lot 452855), citric acid (E-Merck, Germany), sodium lauryl sulphate (E-Merck,Germany), absolute ethanol (EMerck, Germany), potassium bromide (E-Merck,Germany), and demineralized water.

\subsection{Method}

\subsubsection{Preparation of Quercetin-PEG 8000 Solid Dispersion}

Quercetin solid dispersion - PEG 8000 was made with ratio of 1:1; 1:2 and 1:3 (\% w/w). PEG 8000 was placed at the hot plate (Thermolyne Cimarec, USA) and melted at temperature $65-70{ }^{\circ} \mathrm{C}$, then quercetin powder was added. The mixture was stirred until quercetin was dissolved in the melted PEG 8000. The mixture was then cooled to obtain the solid form and dried. The melting temperature of quercetin is around $326^{\circ} \mathrm{C}$. It is assumed that the heating up to around $70^{\circ} \mathrm{C}$ does not affect the stability of quercetin. Afterward the mixture was ground and sieved (50 mesh) to produce a powder. The powder was stored in an airtight container with silica gel.

\subsubsection{Preparation of Physical Mixture (PM) Quersetin-PEG 8000}

The powder of quercetin and PEG 8000 were each sieved through a 50-mesh sieve. The physical mixture was prepared by slowly mixing quercetin - PEG 8000 at a ratio of $1: 1 ; 1: 2$; and $1: 3(\% \mathrm{w} / \mathrm{w})$ until homogeneous in an agate mortar.

\subsubsection{Characterization with Differential Thermal Analyzer (DTA)}

The thermal analysis of the samples was performed using DTA (Mettler Toledo FP90, Switzerland). The temperature was calibrated with indium. A sample of 5-7 $\mathrm{mg}$ was placed on a closed aluminumpan. The DTA was programmed at a temperature range of 50 to $300^{\circ} \mathrm{C}$ with a heating rate of $10^{\circ} \mathrm{C}$ per minute.

\subsubsection{Characterization by Powder X-ray Diffraction Method of (PXRD)}

Powder X-ray diffraction analysis was conducted at room temperature using diffractometer (X'Pert Phillips, Netherlands). The measurement conditions were as follows Cu metal target, Ka filter, $40 \mathrm{kV}$ voltage, $40 \mathrm{~mA}$ current, and the analysis was performed at the range of 2 theta $5-40^{\circ}$. The sample was placed on the sample holder and leveled to prevent the orientation of the particles during sample preparation.

\subsubsection{Characterization with Fourier Transform Infrared Spectroscopy}

A dispersion of about $1 \%$ of a powder sample in potassium bromide $(\mathrm{KBr})$ was prepared by mixing the material with $\mathrm{KBr}$. Infrared spectra were obtained by an infrared spectrophotometer (Spectra One, Perkin Elmer, USA) in the wavenumber range of $400-4000 \mathrm{~cm}^{-1}$. 


\subsubsection{Characterization with Scanning Electron Microscopy (SEM)}

The powder sample was placed on an aluminum holder sample and coated with gold with a thickness of $10 \mathrm{~nm}$. Samples were then observed at various magnifications using SEM device (Jeol, Japan). Voltage and current were set at $20 \mathrm{kV}$ and $12 \mathrm{~mA}$, respectively.

\subsubsection{Solubility Test}

The solubility test of the sample (quersetin, physical mixture and solid dispersion) was carried out at a temperature of $30^{\circ} \mathrm{C} \pm 0.5^{\circ} \mathrm{C}$ by dissolving $20 \mathrm{mg}$ quersetin into $40 \mathrm{~mL}$ of citric acid buffer medium $-\mathrm{NaOH}$ (pH $5.0 \pm 0.5$ ). Citrate buffer (citric acid - NaOH) media with pH $5.0 \pm 0.5$ and temperature $30{ }^{\circ} \mathrm{C}$ was used to maintain the stability of quercetin during dissolution test. The mixture was stirred using magnetic stirrer on water bath circulation (Julabo PURA 22, Germany). Three $\mathrm{mL}$ of sample was taken in 240 minutes. The trailer was then filtered with a $0.45 \mu \mathrm{m}$ Millipore filter membrane. The filtrate was measured using UV-Vis spectrophotometer (Cary 50 Conc, Varian ${ }^{\circledR}$. USA) at a wavelength of $366.95 \mathrm{~nm}$. The solid dispersion solubility was done triplicate.

\subsubsection{In-vitro Dissolution Test}

Dissolution tests were performed on quercetin, physical mixture of quercetin-PEG 8000, and quercetinPEG 8000 solid dispersion equivalent to $5,0 \mathrm{mg}$ of pure quercetin. The dissolution test was carried out using dissolution test apparatus (Erweka DT 700, Germany) with basket mixer. The stirring rate was $100 \mathrm{rpm}$ using the medium of citric acid buffer - NaOH (pH $5.0 \pm 0.5)$ with $1 \%$ SLS surfactant at $37 \pm 0.5^{\circ} \mathrm{C} .5,0 \mathrm{~mL}$ of samplewas taken at $5 ; 10 ; 15 ; 20 ; 25$; and 30 minutes using injection syringes. The sample was filtered with a $0.45 \mu \mathrm{m}$ membrane filter (Millipore, USA) and was determined using the UV-Vis spectrocotometer at a wavelength of $366.95 \mathrm{~nm}$.

Acknowledgments: The authors extend thanks to the Directorate-General of Higher Education, Ministry of Research, Technology, and Higher Education who has provided research funding through Program Unggulan PerguruanTinggi (PUPT) 2015-2016. The authors would like to thank Dr. Chrismawan Ardianto, Department of Clinical Pharmacy Airlangga University, for the valuable discussion and suggestions to improve the manuscript.

Author contributions: Concept -S.D.; Design - S.D., S.R. ; Supervision - S.D, S.R.; Resource - S.F., A.Z.; Materials - S.F., A.Z.; Data Collection and/or Processing - S.F., A.Z.; Analysis and/or Interpretation - S.D.; Literature Search - S.F., A.Z.; Writing - S.R.; Critical Reviews - S.D., S.R.,S.F., A.Z.

Conflict of interest statement: The authors declare no conflict of interest.

\section{REFERENCES}

[1] Sahoo NG, Kakran M, Shaal LA, Li L, Müller RH, Pal M, Tan LP. Preparation and characterization of quercetin nanocrystals. J Pharm Sci. 2011;100(6): 2379-2390.

[2] Madaan K, Lather V, Pandita D. Evaluation of polyamidoamine dendrimers as potential carriers for quercetin, a versatile flavonoid. DrugDeliv. 2016;23(1): 254-262.

[3] Materska M. Quercetin and its derivatives: Chemical structure and bioactivity - a review. Pol J Food Nutr Sci. 2008;58(4): 407- 413.

[4] Li B, Konecke S, Harich K, Wegiel L, Taylor L, S, Edgar KJ. Solid dispersion of quercetin in cellulose derivative matrices influences both solubility and stability. Carbohydr Polym. 2013;92(2): 2033-2040.

[5] Kakran M, Sahoo NG, Li L. Dissolution enhancement of quercetin through nanofabrication, complexation, and solid dispersion. Colloids Surf B Biointerfaces. 2011;88(1): 121-130.

[6] Harwood M, Danielewska-Nikiel B, Borzelleca JF, Flamm GW, Williams GM, Lines TC. A critical review of the data related to the safety of quercetin and lack of evidence of in vivo toxicity, including lack of genotoxic/carcinogenic properties. Food ChemToxicol. 2007;45(11): 2179-2205.

[7] Shah TJ, Amin AF, Parikh JR, Parikh RH. Process optimization and characterization of poloxamer solid dispersions of a poorly water-soluble drug. AAPS PharmSciTech. 2007;8(2): E18-E24.

[8] Verma S, Rudraraju VS. A systematic approach to design and prepare solid dispersions of poorly water-soluble drug. AAPS PharmSciTech. 2014;15(3): 641-657. 
[9] Biswal S, Sahoo J, Murthy PN, Giradkar RP. Enhancement of dissolution rate of gliclazide using solid dispersions with polyethylene glycol 6000. AAPS PharmSciTech. 2008; 9(2): 563-570.

[10] Ho HO, Su HL, Tsai T, Sheu MT. The preparation and characterization of solid dispersions on pellets using a fluidized-bed system. Int J Pharm. 1996;139(1-2): 223-229.

[11] Kalia A, Poddar M. Solid dispersions: an approach towards enhancing dissolution rate. Int J Pharm Pharm Sci. 2011; 3(4): 9-19.

[12] Biswal S, SahooJ, MurthyPN. Characterisation of gliclazide-PEG 8000 solid dispersions. Trop J Pharm Res. 2009; 8(5).

[13] Otto DP, OttoA, De Villiers MM. Experimental and mesoscale computational dynamics studies of the relationship between solubility and release of quercetin from PEG solid dispersions. Int J Pharm. 2013; 456(2): 282-292.

[14] Chuah JN, Talekar M, Gorajana, Garg S. Formulation development and dissolution rate enhancement of efavirenz by solid dispersion systems. Indian J Pharm Sci. 2013 ; 75(3): 291-301.

[15] Karadag A, OzcelikB, HuangQ. Quercetin nanosuspensions produced by high-pressure homogenization. JAgricFoodChem. 2014; 62 (8):1852-1859.

[16] Momić T, Savić J, Černigoj U, Trebše P, Vasić V. Protolytic equilibria and photodegradation of quercetin in aqueous solution. Collect Czech Chem Commun. 2007; 72; 1447-1460.

[17] SerajuddinA. Solid dispersion of poorly water-soluble drugs: Early promises, subsequent problems, and recent breakthroughs. J Pharm Sci. 1999;88(10): 1058-1066.

[18] Leuner C,DressmanJ. Improving drug solubility for oral delivery using solid dispesions. Eur J Pharm Biopharm. 2000;50(1): 47-60.

[19] Singh S, Baghel RS,YadavL. A review on solid dispersion. Int J of Pharm \& Life Sci. 2011; 2(9). 